\title{
The development of the superelement approach applied to models of rod elements
}

\author{
Oleg Kovalchuk* \\ Moscow State University of Civil Engineering, Yaroslavskoe shosse, 26, Moscow, 129337, Russia
}

\begin{abstract}
The article deals with the rod model for the analysis of stressstrain state of rod structures. Analytical solutions are obtained by the operating method. It is assumed that the rod elements can have variables along their axis of physicals characteristics. External loads can also vary along the axes. Calculation formulas are suitable for loads distributed according to an arbitrary law. Unlike the finite element method, defining matrices are formed after obtaining an expression, allowing to calculate the nodal forces through nodal displacements. Examples are given to demonstrate the accuracy of the obtained expressions.
\end{abstract}

\section{Introduction}

For systems of any kind it is true that the disruption of the systems is possible in case of violation of the connectivity of their structures. The system cannot perform its functions when there is no interaction between all or at least vital (key) elements. It is obvious that there is a need to develop analytical and numerical methods for calculating the loaded structural construction systems that can describe their stress-strain state during transients initiated by sudden external influences or internal structural changes. Therefore, we propose a variant of the mathematical model of the rod with arbitrary signs of the distribution of stiffness and density along the axis, which do not contain internal degrees of freedom and its state in any section is determined by the movements of the boundary nodes.

\section{Construction of a mathematical model}

Take the Cartesian coordinate system $\mathrm{x}, \mathrm{y}, \mathrm{z}$ where $\mathrm{x}$ is the longitudinal (axial) coordinate, $y, z$ are the main Central axis of inertia. We assume that the $x, y, z$ axes are combined with the orts of the natural trihedron (Frenet trihedron [6]) t, n, b (tangent, normal, binormal). It is assumed that the deformation of the rod is small in accordance with the operating conditions and Bernoulli hypotheses can be used [7,8]. It is recommended to see the following works $[9,10]$.

A condition that allows to construct a FE model of the rod system is the presence of an expression that allows to calculate the nodal forces through nodal displacements. This is presented in [10-15].

\footnotetext{
* Corresponding author: Kovalhuk@mgsu.ru
} 
As a result, the state at any point of the rod is determined through the nodal displacements.

$$
\begin{aligned}
& \mathbf{y}_{F}(0)=\left\{\mathrm{V}_{F C}^{s t}(0)-\mathrm{V}_{F F}^{s t}(0) \mathrm{V}_{C F}^{s t}(L)^{-1} \mathrm{~V}_{C C}^{s t}(L)\right\} \mathbf{y}_{0 C}+\mathrm{V}_{F F}^{s t}(0) \mathrm{V}_{C F}^{s t}(L)^{-1} \mathbf{y}_{k C}- \\
& -\mathbf{F}_{q F}(0)+\mathrm{V}_{F F}^{s t}(0) \mathrm{V}_{C F}^{s t}(L)^{-1} \mathbf{F}_{q C}(L) ; \\
& \mathbf{y}_{F}(L)=\left\{\mathrm{V}_{F C}^{s t}(L)-\mathrm{V}_{F F}^{s t}(L) \mathrm{V}_{C F}^{s t}(L)^{-1} \mathrm{~V}_{C C}^{s t}(L)\right\} \mathbf{y}_{0 C}+\mathrm{V}_{F F}^{s t}(L) \mathrm{V}_{C F}^{s t}(L)^{-1} \mathbf{y}_{k C}- \\
& -\mathbf{F}_{q F}(L)+\mathrm{V}_{F F}^{s t}(L) \mathrm{V}_{C F}^{s t}(L)^{-1} \mathbf{F}_{q C}(L)
\end{aligned}
$$

Here $\mathbf{F}_{q}$ is the vector of nodal forces equivalent to the distributed load, $\mathbf{V}-$ this is the matrix of influence of initial parameters, built on the basis of the analytical solution of the equations of state. [10-15] The influence matrix is a property of the rod that does not depend either on external loads or on the conditions of fastening the rod.

We combine nodal moments and forces into a vector of nodal force factors:

$$
\mathbf{F}_{\text {node }}=\left\{\mathbf{y}_{F}(0) \quad \mathbf{y}_{F}(L)\right\}
$$

Similarly we proceed with the displacements of the ends of the rod. We obtain the vector of nodal displacements:

$$
\mathbf{U}_{\text {node }}=\left\{\mathbf{y}_{0 C}(0) \quad \mathbf{y}_{k C}\right\}
$$

Then (1) can be written compactly:

$$
\mathbf{F}_{\text {node }}=\mathbf{K U}_{\text {node }}-\mathbf{F}_{q}^{\text {node }}
$$

where we have introduced the stiffness matrix of the rod:

$$
\mathrm{K}=\left[\begin{array}{cc}
\mathrm{V}_{F C}^{s t}(0)-\mathrm{V}_{F F}^{s t}(0) \mathrm{V}_{C F}^{s t}(L)^{-1} \mathrm{~V}_{C C}^{s t}(L) & \mathrm{V}_{F F}^{s t}(0) \mathrm{V}_{C F}^{s t}(L)^{-1} \\
\mathrm{~V}_{F C}^{s t}(L)-\mathrm{V}_{F F}^{s t}(L) \mathrm{V}_{C F}^{s t}(L)^{-1} \mathrm{~V}_{C C}^{s t}(L) & \mathrm{V}_{F F}^{s t}(L) \mathrm{V}_{C F}^{s t}(L)^{-1}
\end{array}\right]
$$

and the vector of nodal loads from external loads on the rod:

$$
\mathbf{F}_{q}^{\text {node }}=\left\{\mathbf{F}_{q F}(0)+\mathrm{V}_{F F}^{s t}(0) \mathrm{V}_{C F}^{s t}(L)^{-1} \mathbf{F}_{q C}(L) \quad \mathbf{F}_{q F}(L)+\mathrm{V}_{F F}^{s t}(L) \mathrm{V}_{C F}^{s t}(L)^{-1} \mathbf{F}_{q C}(L)\right\}
$$

Expression (4) by forms, like the analogous FEM equation in the form of the displacement approach. Using the terminology of FEM (MSE), the result is an expression for one structural element of the system - the continuum superelement of the rod (KSER). Its initial relations do not contain internal degrees of freedom and its state in any section is determined by the displacements of the boundary nodes. He models an element of the subsystem - one rod.

\section{Verification of the obtained relationships}

The correctness of mathematical modeling made in the formulation of the relations (1) is confirmed by constructing the calculation formulas for dimensionless components of the state under static loading of the rod (Fig. 1). It is a rod has known length $L$, Young's 
modulus $E$, cross-sectional area $A$, principal central moments of inertia $J_{y}, J_{z}$ from dimensionless longitudinal coordinate $\xi=x / L$ :

$$
\begin{aligned}
& u=\frac{u_{x}}{L} ; v=\frac{u_{y}}{L} ; w=\frac{u_{z}}{L} ; n=\frac{N}{E A} ; \Theta_{y}=\frac{L^{2} Q_{y}}{E J_{z}} ; \Theta_{z}=\frac{L^{2} Q_{z}}{E J_{y}} ; \\
& m_{x}=\frac{L M_{x}}{G\left(J_{y}+J_{z}\right)} ; m_{y}=\frac{L M_{y}}{E J_{y}} ; m_{z}=\frac{L M_{z}}{E J_{z}} . \\
& \mu_{x}(\xi, t)=\frac{L^{2} m_{x}(\xi, t)}{G\left(J_{y}+J_{z}\right)} ; f_{x}=\frac{L q_{x}}{E A} ; f_{y}=\frac{L^{3} q_{y}}{E J_{z}} ; f_{z}=\frac{L^{3} q_{z}}{E J_{y}}
\end{aligned}
$$

Dimensionless longitudinal external force and external bending moment:

$$
p=\frac{P}{E A} ; m=\frac{M L}{E J_{y}}
$$

$u_{x}=0 ; u_{y}=0 ; u_{z}=0$;

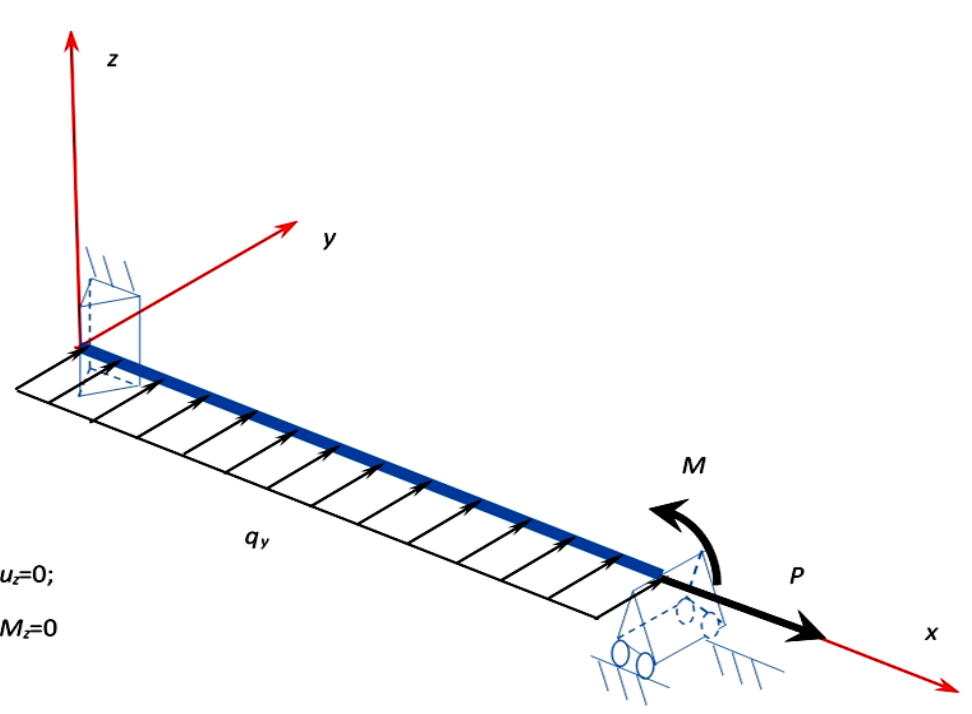

$\theta_{\mathrm{x}}=0 ; \quad \theta_{y}=0 ; \quad M_{z}=0$

$N=P ; u_{y}=0 ; u_{z}=0$;

$\theta_{x}=0 ; M_{y}=M ; \quad \theta_{2}=0$

Fig. 1. Boundary conditions and external loads are given.

Table 1. The dimensionless factors and calculation formulas for Fig. 1.

\begin{tabular}{|c|c|}
\hline The dimensionless factor & Calculation formula \\
\hline The longitudinal movement of the & $p \xi$ \\
\hline Deflection axis $y$ & $\frac{f_{y}}{48} \xi(\xi-1)^{2}(2 \xi+1)$ \\
\hline Deflection axis $z$ & $\frac{m}{4} \xi^{2}(\xi-1)$ \\
\hline
\end{tabular}




\begin{tabular}{|c|c|}
\hline The dimensionless factor & Calculation formula \\
\hline Angle of twist & 0 \\
\hline $\begin{array}{c}\text { The rotation angle of the cross section } \\
\text { relative to the axis } y\end{array}$ & $\frac{m}{4} \xi^{2}(3 \xi-2)$ \\
\hline $\begin{array}{c}\text { The rotation angle of the cross section } \\
\text { relative to the axis } z\end{array}$ & $\frac{f_{y}}{48}\left(8 \xi^{3}-9 \xi+1\right)$ \\
\hline Torsional moment & 0 \\
\hline The bending moment in the plane $x z$ & $-\frac{m}{2}(3 \xi-1)$ \\
\hline The bending moment in the plane $x z$ & $-\frac{f_{y}}{8} \xi(4 \xi-3)$ \\
\hline Longitudinal force & $-\frac{f_{y}}{8}(8 \xi-3)$ \\
\hline Transverse force along the axis $y$ & $-\frac{3}{2} m$ \\
\hline Transverse force along the axis $z$ & \\
\hline
\end{tabular}

Below, Fig. 2, 3 shows graphs of changes by the length of the rod dimensionless factors. To match the scales in the graphs of dimensionless displacements and longitudinal and transverse forces, the parameter $p$-dimensionless longitudinal force - was taken to be 0.05 ; the parameter $f_{y}$ was taken to be one.
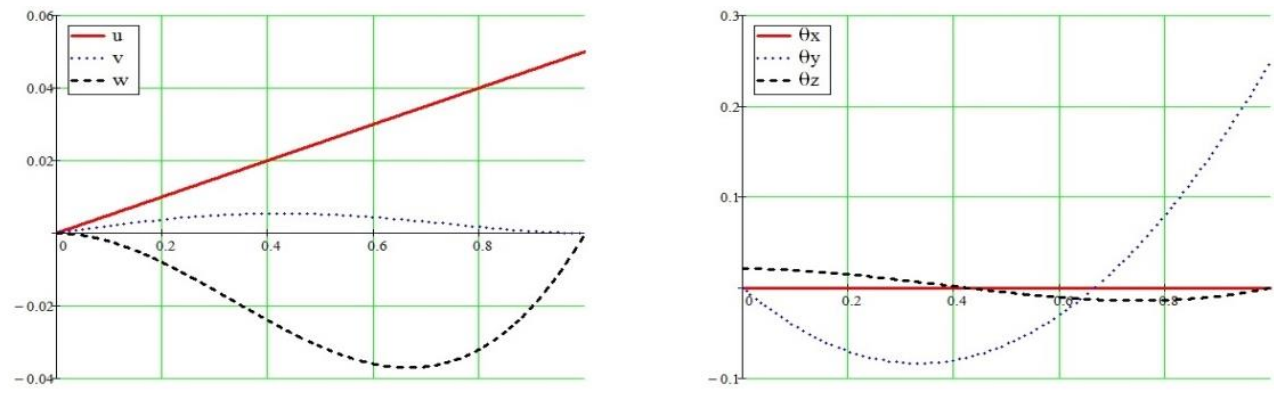

Fig. 2. Dimensionless displacement and the angles of twist and turn.
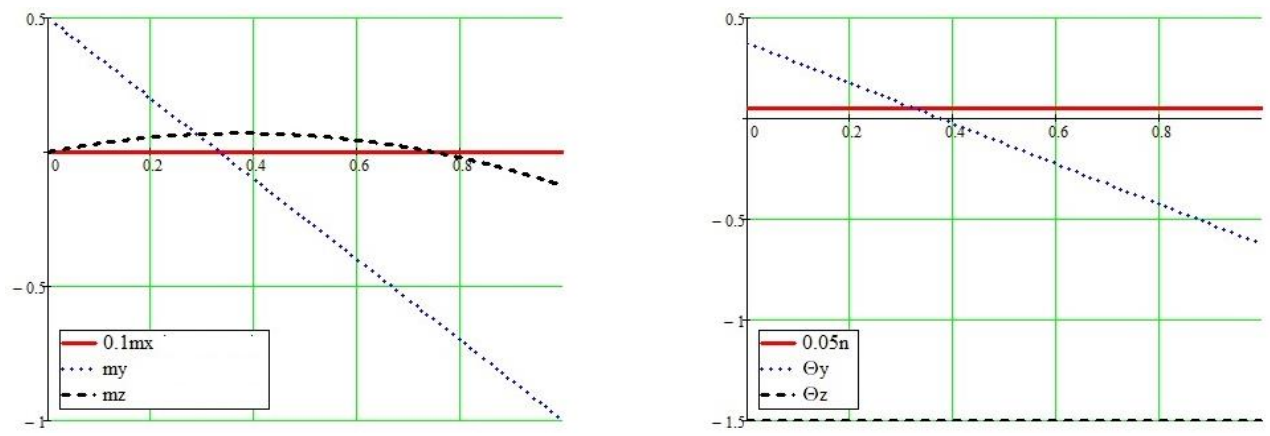

Fig. 3. Dimensionless moments and dimensionless longitudinal and transverse forces. 
From Fig. 1-3 it can be seen that both the force and kinematic conditions are satisfied (which, however, is obvious from the calculation formulas). Thus, at least for statics, the formula (1) is satisfied.

To check the correctness of the same formula in the dynamic calculation, a dynamic stiffness matrix was constructed and a homogeneous dynamic problem (on free vibrations of the rod) under different boundary conditions was considered. Frequency equations representing the dependence of the main determinant of the stiffness matrix with imposed kinematic conditions from dimensionless quantities were compared $\omega k_{\text {ten }}, \omega k_{\text {tor }}$, $\sqrt{\omega} k_{y}, \sqrt{\omega} k_{z}$, where

$$
k_{\text {ten }}^{2}=L^{2} \frac{\rho}{E} ; k_{\text {tor }}^{2}=L^{2} \frac{\rho}{G} ; k_{y}^{4}=L^{4} \frac{\rho A}{E J_{y}} ; k_{z}^{4}=L^{4} \frac{\rho A}{E J_{z}}
$$

Table 2. The frequency equations for different variants the end restraint.

\begin{tabular}{|c|c|}
\hline Conditions of fastening & The frequency equation \\
\hline $\begin{array}{c}\text { The swivel bearing at the } \\
\text { beginning and end, bending in } \\
\text { the plane } x y\end{array}$ & $\frac{2 \omega k_{z}^{2} \operatorname{sh}\left(\sqrt{\omega} k_{z}\right) \sin \left(\sqrt{\omega} k_{z}\right)}{\operatorname{ch}\left(\sqrt{\omega} k_{z}\right) \cos \left(\sqrt{\omega} k_{z}\right)-1}=0$ \\
\hline $\begin{array}{c}\text { Built-in support in the beginning, } \\
\text { freely at the end, spatial bending } \\
\text { with stretching and twisting }\end{array}$ & $\frac{\omega^{6} k_{y}^{4} k_{z}^{4} k_{\text {ten }} k_{\text {tor }} \operatorname{ctn}\left(\omega k_{\text {ten }}\right) \operatorname{ctn}\left(\omega k_{\text {tor }}\right) \cdot}{\left(\cos \left(\sqrt{\omega} k_{y}\right) \operatorname{ch}\left(\sqrt{\omega} k_{y}\right)+1\right)\left(\cos \left(\sqrt{\omega} k_{z}\right) \operatorname{ch}\left(\sqrt{\omega} k_{z}\right)+1\right)}$ \\
\hline $\begin{array}{c}\text { Built-in support in the end, freely } \\
\text { at the beginning, spatial bending } \\
\text { with stretching and twisting }\end{array}$ & $\frac{\omega^{6} k_{y}^{4} k_{z}^{4} k_{\text {ten }} k_{t o r} \operatorname{ctn}\left(\omega k_{\text {ten }}\right) \operatorname{ctn}\left(\omega k_{t o r}\right) \cdot}{\left(\cos \left(\sqrt{\omega} k_{y}\right) \operatorname{ch}\left(\sqrt{\omega} k_{y}\right)+1\right)\left(\cos \left(\sqrt{\omega} k_{z}\right) \operatorname{ch}\left(\sqrt{\omega} k_{z}\right)+1\right)}$ \\
\hline
\end{tabular}

Note that the formulas in the second and third lines should coincide in essence with the problems, since for the natural frequencies it does not matter whether the end or the beginning is clamped. Further, the numerators of the formulas coincide with those given in the Handbook of Birger et al. (v. 3) for hinged and cantilever rods. [16] The presence of the denominators is a consequence of the matrix inversion $\mathrm{V}_{C F}$ in equation (5), however, the excess of roots at the same is not added. The same can be said about the frequencies of stretching and torsion, if you express the cotangent through the sine and cosine. The presence of possible zeros in the denominators is not critical. It is easy to avoid its influence by replacing the solution of the frequency equation with the minimization of its square: then the break points will give maximum, and the roots - minimum.

\section{Conclusions}

The solution based on analytical formulas allows to determine the behavior of the system or its element under different external influences. The proposed method of modeling the rod as a continuum spatial superelement for static and dynamic problems is original. The calculation of the system of such finite elements in the form of FEM is of practical 
importance. Calculations are possible for statically definable and statically indeterminate systems.

\section{References}

1. V.A. Postnov, S.P. Dmitriev, Method of Superelements in the Calculation of Engineering Structures (Shipbuilding, Leningrad, 1979)

2. Y.N. Ogurtsov, Structural mechanics and calculation of constructions J., 5, 50 (1989)

3. V.I. Pletnev, M.V. Sergeev, Construction and architecture J., 10, 116 (1998)

4. V.A. Postnov, Numerical methods for calculation of ship structures (Shipbuilding, Leningrad, 1977)

5. E.S. Przheminitsky, Rocketry and Astronautics J., 1, 165 (1963)

6. L.A. Tolokonnikov, Mechanics of deformable solids, (Higher school, Moscow, 1979)

7. S. M. Han, H. Benaroya, T. Wei, Journal of Sound and Vibration, 5, 935 (1999)

8. S.P. Timoshenko, J.M. Gere, Theory of elastic stability (McGraw-Hill, New York, 1961)

9. V.A. Gordon, A.G. Tamrazyan, T.V. Savostikova, National Research Center Bulletin 2, 167 (2010)

10. A.G. Tamrazyan, O.A. Kovalchuk, National Research Center Bulletin, 3-4, 120 (2011)

11. O.A. Kovalchuk, Procedia Engineering J., 153, 304 (2016)

12. O.A. Kovalchuk, MATEC Web of Conferences, 86, (2016)

13. O.A. Kovalchuk, Industrial and Civil Engineering J., 8, 70 (2016)

14. O.A. Kovalchuk, Natural and technical Sciences J., 11-12, 344 (2014)

15. O.A. Kovalchuk, Building: Science and Education J., 1, 1 (2012)

16. I.A. Birger, Y.G. Panovko, Strength, Stability, Vibrations (Mechanical Engineering, Moscow, 1968) 